\title{
Research on Prediction of Ground Settlement of Deep Foundation Pit Based on Improved PSO-BP Neural Network
}

\author{
Liu Yuhao ${ }^{1, *}$, Feng Xiao ${ }^{2, b}$ \\ ${ }^{1}$ School of Civil Engineering, Chongqing Jiaotong University, 400074 Chongqing, China \\ ${ }^{2}$ School of Civil Engineering, Chongqing Jiaotong University, 400074 Chongqing, China
}

\begin{abstract}
In view of the limitations of the existing prediction methods for ground subsidence of deep foundation pit, a BP neural network prediction model based on improved particle swarm optimization algorithm was proposed. The mutation and crossover of genetic algorithm are integrated into particle swarm optimization algorithm, which makes full use of the global characteristics of genetic algorithm and the fast convergence speed of particle swarm optimization algorithm. In order to reduce the network output error, improve the convergence speed and enhance the network generalization ability, the final value of the optimized particle iteration was selected as the connection weight and threshold of the BP neural network. The results show that the RMSE, MAPE and $\mathrm{R}^{2}$ of the improved PSO-BP model are $0.3077,0.7506 \%$ and 0.8811 , so the improved PSO-BP model has a better prediction accuracy.
\end{abstract}

\section{Introduction}

With the rapid development of urban underground space construction, the excavation area and depth of deep foundation pit increase gradually, and the settlement of soil around deep foundation pit will occur in the excavation process. In serious cases, ground subsidence and deformation will greatly affect the safety of buildings, underground structures and pipelines around deep foundation pits.

In view of the uncertainty and fuziness of many influencing factors of deep foundation pit surface settlement, as well as the nonlinear characteristics of deep foundation pit surface settlement, many scholars used BP neural network to predict the deformation of deep foundation pit. BP neural network has the characteristics of large scale of parallel data processing, strong robustness, high degree of nonlinear fitting and strong learning ability, etc. [1], which is suitable for the prediction of deep foundation pit surface settlement. Fang, et al [2]. Based on the grey system theory and artificial neural network theory, the grey GM $(1,1)$ model, the BP neural network model and the grey BP neural network combination model are used to predict the ground subsidence of deep underground foundation pit. According to different working conditions, $\mathrm{Li}$ et al. [3]. Taking the monitoring data as input, the BP neural network model is established to predict the horizontal displacement of piles, and the prediction results have good adaptability. Liang et al [4]. used wavelet threshold denoising method to denoise the actual observation data of longitudinal observation line. According to the principle of the highest SNR and the minimum root mean square, a prediction model of foundation pit deformation combining wavelet transform and BP neural network is put forward, and good results are achieved.

Many scholars [5] use particle swarm optimization algorithm (PSO) to optimize the BP neural network, and the formed network model has good performance. However, the standard particle swarm optimization algorithm has problems such as slow convergence speed, local optimization and premature convergence, which lead to inaccurate weight selection. In order to prevent particles from falling into the local optimal solution and ensure that the particle diversity converges to the global optimal solution as soon as possible, our team adjusted the learning factors and inertia weights, and used genetic algorithms to improve the particle swarm optimization algorithm. Finally, an improved particle swarm optimization algorithm is used to select the weights and thresholds of the BP neural network.

\section{Improved particle swarm optimization back propagation neural network}

\subsection{Back propagation neural network}

The structure of BP neural network includes input layer, hidden layer and output layer. BP neural network has the following characteristics:

The network is a multi-layer structure. Each neuron in the adjacent layer is connected to all neurons in the adjacent layer. This network structure enables BP neural network to complete complex computation.

The activation function of the network is differentiable, such as the commonly used Sigmoid function and linear function. According to the mapping

\footnotetext{
*Corresponding author: ${ }^{\mathrm{a} 1457251985 @ q q . c o m}$

b794263718@qq.com
} 
interval range, the Sigmoid function can be further divided into log-sigmoid function and tan-sigmoid function, as shown in Formula 1.

$$
\left\{\begin{array}{c}
f(x)=\frac{1}{1+e^{-x}} \\
f(x)=\frac{1}{1+e^{-2 x}-1}
\end{array}\right.
$$

In Formula 1, the value of $\mathrm{x}$ is any real number, and the output interval of the function is $[0,1]$ and $[-1,1]$.

Back-Propagation Algorithm is adopted in the network. The principle of BP algorithm is that in supervised learning, the mean square value of the difference between the actual output and the expected output can be used as an error signal to propagate backwards along the network. During the propagation process, the weight of each layer of the network will be adjusted. This process will be repeated until the error is lower than the target value and the learning of the neural network ends. This algorithm can effectively train the multi-layer forward neural network with excellent nonlinear fitting ability, so this algorithm has been widely paid attention to by researchers for many years [6]

\subsection{Particle swarm optimization algorithm}

Particle Swarm Optimization algorithm is based on the natural phenomenon of birds foraging. At the beginning of foraging, each bird's position is independent, and the foraging direction is random and uncertain. Each bird is optimized based on the location of the bird closest to the food in the system and its subsequent foraging direction. Particle Swarm Optimization (PSO) initializes a group of random particles in the search space and obtains the optimal solution through iteration. Suppose there are $\mathrm{m}$ particle clusters in the d-dimensional target search space, and the vector $X_{i}=\left(x_{i 1}, x_{i 2}, \ldots, x_{i d}, \ldots, x_{i D}\right)$ is the position of the particle, and vector $V_{i}=\left(v_{i l}, v_{i 2}, \ldots, v_{i d}, \ldots, v_{i D}\right)$ is speed. In the iteration process, the particle will constantly update the individual optimal solution Pbest and the global optimal solution Gbest [7]. Update the velocity and position of each particle according to the following formula:

$$
\begin{aligned}
& v_{i d}^{k+1}=w(k) v_{i d}^{k}+c_{1} r_{1}^{k}\left(p_{i d}-x_{i d}^{k}\right)+c_{2} r_{2}^{k}\left(p_{g d}-x_{i d}^{k}\right) \\
& x_{i d}^{k+1}=x_{i d}^{k}+v_{i d}^{k+1}
\end{aligned}
$$

In Formula 2 and $3, k$ is the number of current iterations; $v_{i d}^{k}$ and $v_{i d}^{k+1}$ are the velocities of particle $i$ at $k$ and $k+1$ iterations in the d-dimensional component, respectively. $x_{i d}^{k+1}$ and $x_{i d}^{k}$ are the positions of particle $i$ in the d-dimensional component for $k$ and $k+1$ iterations, respectively. $c_{1}$ and $c_{2}$ are non-negative constant learning factors. $r_{1}$ and $r_{2}$ are random numbers in the range $[0,1] ; w(k)$ is the inertia weight coefficient; $v_{i d} \in\left[-v_{\max }, v_{\max }\right]$, Where $V_{\max }$ is a constant used to prevent particles from escaping from the solution space.

\subsection{Improved particle swarm optimization algorithm}

The standard BP neural network has poor learning stability and low approximation accuracy for the connection weights of structural elements. At the same time,PSO algorithm is easy to fall into the local optimal solution. There are many improvements in particle swarm optimization algorithm. The inertia weight reflects the ability of the particle to inherit the speed of the last iteration. Therefore, inertia weight can be used as a mechanism to control group search ability. Formula 4 is used in this paper to optimize the linear decline weight[8].

$w_{k}=w_{\max }-\frac{k\left(w_{\max }-w_{\min }\right)}{N}$

In Formula $4, w_{\min }$ and $w_{\max }$ are the minimum and maximum inertial weights respectively. They are updated based on the results of each iteration. $N$ is the maximum number of iterations and $k$ is the current number of iterations.

In order to obtain the diversity of particles in the initial search stage and converge to the global optimal solution as soon as possible, in the subsequent stage, through the analysis of the influence of the change factors of learning factors, $c_{1}$ and $c_{2}$ can better balance the global and local search after dynamic adjustment and optimization by using tangent function. The tangent function is expressed as follows:

$$
\begin{aligned}
& c_{1}(t)=\left(c_{11_{-} \text {start }}-c_{1_{\_} \text {end }}\right) \times \tan \left(0.875 \times\left(1-\left(\frac{t}{N}\right)^{0.6}\right)\right)+c_{1 \_ \text {end }} \\
& c_{2}(t)=\left(c_{2_{-} \text {start }}-c_{2_{\text {_end }}}\right) \times \arctan \left(2.755 \times\left(1-\left(\frac{t}{N}\right)^{0.4}\right)\right)+c_{2_{-} \text {end }}
\end{aligned}
$$

At the initial stage of search, $c_{1}$ is greater than $c_{2}$, and each particle pays attention to its own historical information to ensure diversity. But in anaphase, $c_{1}$ goes down, $c_{2}$ goes up. The particle pays more attention to the overall information of the group and maintains rapid convergence.

In the early stage of optimization search, PSO algorithm generally shows a fast convergence rate, and all particles are close to the optimal position, which leads to the unification of particles and slows down the convergence rate in the later stage of optimization search. Therefore, the crossover and mutation operations of GA algorithm are introduced, and the global optimization characteristics of GA algorithm are combined to improve the convergence speed of particles in the local region, so as to enhance the particle search ability and realize the complementarity of the two algorithms. First, crossover operation is conducted on particles, and crossover is carried out according to a certain probability. For particles paired with $X_{i}$ and $X_{j}$, the crossover process of position and velocity is as follows:

$$
\begin{array}{r}
\left\{\begin{array}{l}
X_{i}^{k+1}=\alpha_{1} \times X_{i}^{k}+\left(1-\alpha_{1}\right) X_{j}^{k} \\
X_{j}^{k+1}=\left(1-\alpha_{1}\right) \times X_{i}^{k}+\alpha_{1} X_{j}^{k}
\end{array}\right. \\
\left\{\begin{array}{c}
V_{i}^{k+1}=\alpha_{2} \times V_{i}^{k}+\left(1-\alpha_{2}\right) X_{j}^{k} \\
V_{j}^{k+1}=\left(1-\alpha_{2}\right) \times V_{i}^{k}+\alpha_{2} X_{j}^{k}
\end{array}\right.
\end{array}
$$

The new individual obtained by mutation of the particle with a certain probability after crossover operation of position and velocity:

$$
\begin{aligned}
& X_{i j}= \begin{cases}X_{i j}+\left(X_{i j}-X_{\max }\right) \times f(g) & r_{1} \leq 0.5 \\
X_{i j}+\left(X_{\min }-X_{i j}\right) \times f(g) & \mathrm{r}_{1}<0.5\end{cases} \\
& f(g)=r_{2}\left(1-k / G_{\max }\right)
\end{aligned}
$$


In Equations 9 and 10, $X_{\max }$ and $X_{\min }$ are the upper and lower bounds of the value of particle $X_{i j}$ respectively, and Gmax is the maximum evolution algebra.

The specific steps of optimizing BP neural network by improved particle swarm optimization algorithm are shown in Fig. 1.

The root mean square error (RMSE) of each network was taken as the approximate fitness function for calculating the fitness value, namely Formula 11. According to the fitness function $f(x)=E(x)$, the minimum error value $E_{\min }$ was calculated as the particle screening threshold.

$$
\operatorname{RMSE}\left(y_{i}^{\prime}\right)=\sqrt{\frac{1}{n} \sum_{i=1}^{n}\left(y_{i}-y_{i}^{\prime}\right)^{2}}
$$

In formula $11, y_{i}$ and $y_{i}^{\prime}$ are the target value and the predicted value respectively.

Step 1: The BP neural network is initialized, that is, the number of neurons in each layer, and the data is preprocessed.

Step 2: Initialize each parameter in the particle swarm, namely, the inertial weights $w_{\min }$ and $w_{\max }$, and the learning factors $c_{1}$ and $c_{2}$.

Step 3: Update inertial weight and particle velocity and position.

Step 4: Evaluate the historical best position of each particle.

Step 5: Update the global best location.

Step 6: GA algorithm is used to cross and mutate the particles.

Step 7: Continue to update $v_{i}$ of particles under different components. If the training error is less than $E_{\min }$ or the number of iterations reaches $t_{\max }$, the next step will be entered.Otherwise, go back to step 3 .

Step 8: The optimal value after PSO optimization is used as the initial weight and threshold of BP neural network.The BP neural network is pretrained, and the modeling of BP neural network is finally completed.

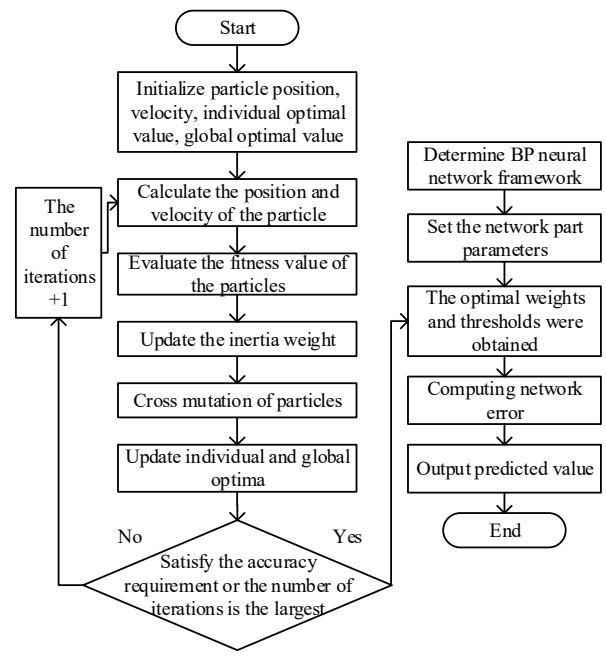

Fig. 1. Improved flow chart of PSO-BP model

\section{Experimental verification}

In this paper, the 315-day monitoring data from the ground D4 settlement observation point of a deep foundation pit on February 2, 2019 solstice and
December 2, 2019 solstice were used as data samples for prediction. The observation point is blocked due to the influence of foundation pit construction. By preprocessing the data, an isometric time series dataset with a time interval step of $24 \mathrm{~h}$ is obtained.

On Matlab2018b platform, using Python language and MySGL database, the simulation experiment of surrounding rock deformation value prediction based on BP neural network is carried out. In this paper, the surface monitoring data of the foundation pit are pretreated, and five influencing factors, such as number of foundation pit support, excavation days, excavation depth, permeability coefficient and cohesion, are taken as input variables of the divine net. The mapminmax function is used to normalize the sample input data into [$1,1]$, and the ratio of $7: 2: 1$ is adopted to form the training sample set, the verification sample set and the test sample set.

The number of hidden layer neurons is very important to BP neural network. In the hidden layer, the number of neurons is mostly set according to empirical formulas. According to the empirical formula, the number range of neurons is determined as $[4,13]$. The PSO-BP neural network model is tested repeatedly, and the optimal parameters obtained in the test process are used for evaluation and prediction. By comparing network errors, the optimal number of nodes in the hidden layer is determined to be 13. The structure of PSO-BP neural network is determined to be 5-13-4. Set the number of iteration termination as 4000 , the learning rate as 0.01 , and the target error as $1 \times 10^{-6}$.

The parameters of the particle swarm optimization algorithm are set as $c_{1}=c_{2}=1.49445$, where the number of particles is 30 . The number of particle iterations is 50 , the particle velocity range is $[-1,1]$, and the particle position range is $[-5,5]$. The improved PSO-BP neural network was trained with parameters set as follows : $c_{1_{\text {_start }}}=2.9, c_{1_{\text {_end }}}=0.78, c_{2_{\text {_t tart }}}=1, c_{2_{\text {_end }}}=3, w_{\text {min }}=0.5$, $w_{\max }=1$. The experimental results of the three models are shown in Figure 2 and Figure 3. The experimental results show that the improved PSO-BP model proposed in this paper is suitable for the analysis and processing of nonlinear deformation sequence, and can effectively predict the ground subsidence of deep foundation pit with good accuracy. In addition, the predicted step sizes of 6 and 8 were selected in this paper to further verify the model. Similarly, the improved PSO-BP model also achieved a good prediction effect in the experiment with the prediction step size of 6 and 8 , respectively. The experimental results show that the improved PSO-BP model can well reflect the variation law of ground subsidence in deep foundation pit. As shown in Fig. 4, for the PSO algorithm, the fitness value of the modified PSO algorithm converges faster, the minimum value is lower, and the weight of the output BP neural network is more appropriate. In order to further verify the effectiveness of the new algorithm, the mean absolute error (MAPE), root mean square error (RMSE) and $\mathrm{R}^{2}$ were used to evaluate the prediction accuracy of each model. The prediction accuracy evaluation of the three models is shown in Table 1. Compared with the other two models, the prediction accuracy of the improved 
PSO-BP model has been significantly improved in all aspects.

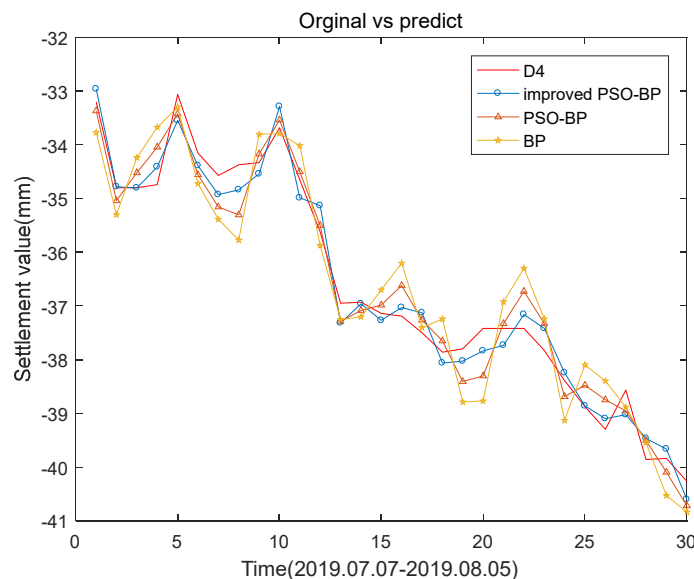

Fig. 2. Surface subsidence prediction curve

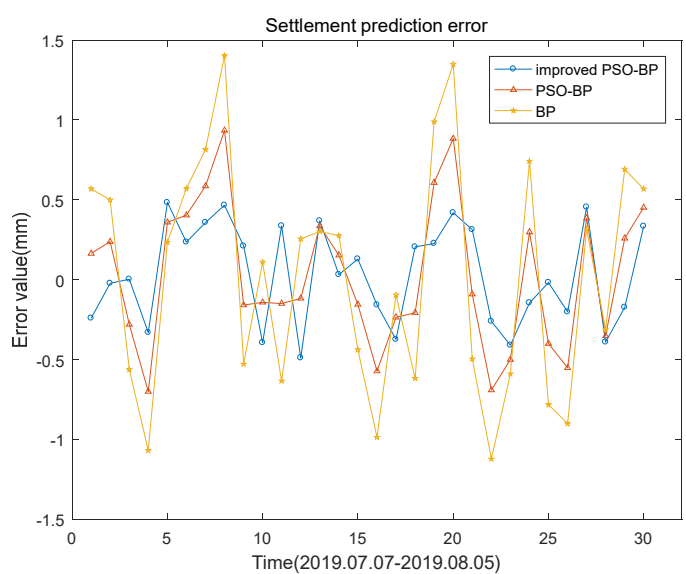

Fig. 3. Prediction error curve of surface subsidence

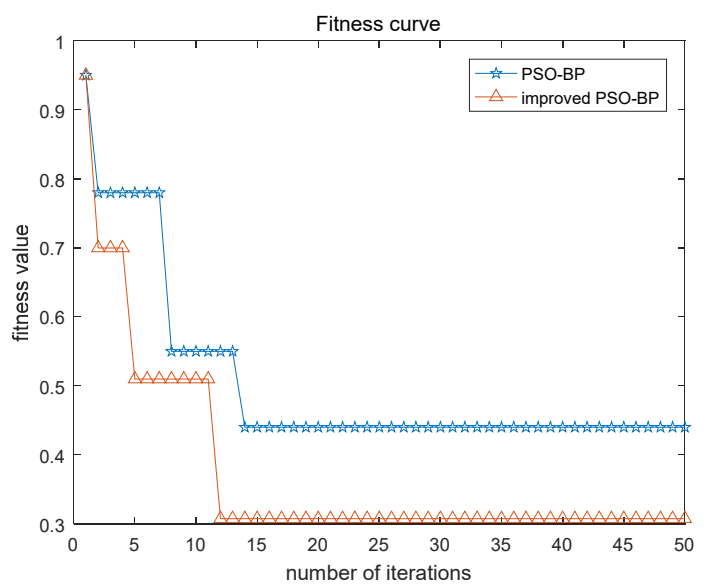

Fig. 4. Fitness value curve

\section{Conclusion}

On the basis of modeling and analysis of actual engineering monitoring data, the monitoring data of deep foundation pit surface settlement are verified and analyzed by multi-step prediction method, aiming at the characteristics that the monitoring values of deep foundation pit surface settlement are nonlinear sequence. By changing the inertia weights and learning factors in particle swarm optimization (PSO) algorithm, the concepts of crossover and mutation in genetic algorithm were introduced into particle swarm optimization (PSO) algorithm. The initial weights and thresholds of BP neural network were optimized, and an improved PSOBP neural network prediction model was proposed. Experimental results show that, compared with PSO-BP model, the proposed algorithm has better prediction effect, higher prediction accuracy and higher stability.

Table 1. Forecast statistics of surface subsidence.

\begin{tabular}{|c|c|c|c|}
\hline & RMSE/mm & MAPE/\% & $\mathrm{R}^{2}$ \\
\hline BP & 0.7106 & 1.7151 & 0.8031 \\
\hline PSO-BP & 0.4401 & 1.0338 & 0.8619 \\
\hline $\begin{array}{c}\text { Improved } \\
\text { PSO-BP }\end{array}$ & 0.3077 & 0.7506 & 0.8811 \\
\hline
\end{tabular}

\section{References}

1. Wu, W., Wang, J., Cheng, M.S. (2011) Convergence analysis of online gradient method for BP neural networks. Neural Networks, 24: 91-98.

2. Fang, L.S., He, G.W., Luo, C.M. (2017) Application of grey theory and BP neural network theory in deformation prediction of subway deep foundation pit. The construction technology, 46: 57-60.

3. Li, S., Li, H., Xia, Y.W. (2017) Application of BP neural network in foundation pit deformation prediction. Roads and motor transport, 182 : 97-101.

4. Liang, X.L., Qi, E.H., Wang, Q.K. (2021) Study on wavelet denoising and BP neural network combined prediction model of foundation pit deformation. Surveying and Mapping and Spatial Geographic Information, 44: 189-192+195.

5. Miyatake, M., Veerachary, M., Toriumi, F. (2011) Maximum power point tracking of multiple photovoltaic arrays: a PSO approach. Ieee Transactions on Aerospace and Electronic Systems, 47: $367-380$

6. Qi, Q., Chen, F.F., Zhao, H. (2021) Short-term prediction of photovoltaic output based on optimized BP neural network. Computer measurement and control, 29: 70-75.

7. Huang, Y., Xiang, Y.X., Zhao, R.X. (2020) Air Quality Prediction Using Improved PSO-BP Neural Network. Ieee Access, 8: 99346-99353.

8. Dan, B.M., Xue, Y.J. (2021) Prediction of blast furnace coal injection volume based on improved PSO optimized ELM. Electronic measurement technique, 44: 93-98. 